\title{
Chapter 22 \\ A Way Forward: The Future of Cancer Health Disparities Research in Latinos
}

\author{
Amelie G. Ramirez and Edward J. Trapido
}

The vision for Advancing the Science of Cancer in Latinos was to bring together researchers, scientists, physicians, health care professionals, patient advocates, and students from across the nation and beyond, engaging them in open dialogue to summarize research advancements to date, identify gaps, and develop actionable goals to translate basic research into clinical best practices, effective community interventions, and professional training programs to eliminate cancer disparities in Latinos.

This conference not only brought together a diverse group of researchers, but it also built upon the pioneering work of Redes En Acción: The National Latino Cancer Research Network (Redes). The Institute for Health Promotion Research established the long-standing network in early 2000 under the National Cancer Institute's (NCI) Center to Reduce Cancer Health Disparities (formerly the Special Populations Networks program). Nineteen years later, Redes maintains collaboration with six regional sites (San Antonio, San Francisco, San Diego, Houston, Miami, and New York) with a combined network of more than 2000 community leaders, researchers, government health agencies, and public advocates, from across the nation dedicated to fighting cancer among Latinos through research, training, and awareness.

Early on, the Redes network identified cancer issues of greatest relevance to Latinos. These identified issues laid the foundation for a national Latino cancer agenda, providing a useful tool for individuals and organizations engaged in cancer prevention and control efforts among Latino populations [1-4]. The Advancing the Science of Cancer in Latinos conference provided a pulse check to see what has

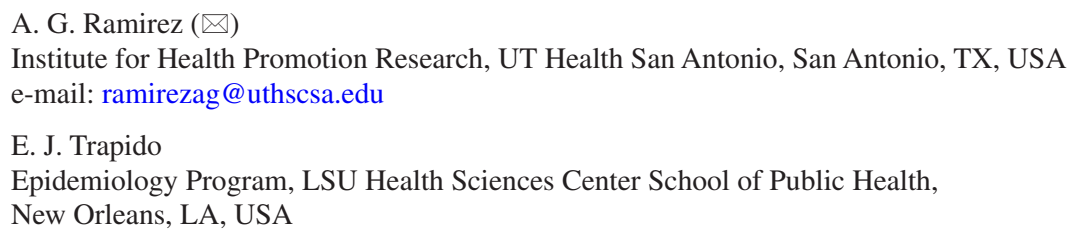


been accomplished, what has been learned, what is still unknown, and what is next. Toward that end, most of the conference presenters made recommendations for a way forward; some of the suggestions are specific to their field of study and others are intended to be applied more broadly. The following are some key recommendations abstracted from their conference presentations.

\section{Genetics, Environment, Lifestyle, and Cancer}

- Cancer prevention strategies are needed now to address changing demographics in the United States. The US Latino population is growing and is projected to double in the next 40 years. Because US Latinos are a young demographic whose cancer burden will rise as they age, there is an urgent need for prevention strategies now, such as promoting cancer screening and altering modifiable risk factors (Chap. 2; Pinheiro, Callahan, and Kobetz; Chap. 21; Rios).

- Disaggregate Latino cancer data into subgroups. The US Latino population is a heterogeneous mix of subgroups that may differ in country of origin, acculturation, nativity, socioeconomic status, and US region in which they reside; these factors can influence cancer risk and outcome. Most US cancer data reports on Latinos as an aggregate group, obscuring the differences that exist among subgroups (Chap. 2: Pinheiro, Callahan, and Kobetz).

- Improve data collection on Latino national origin so that cancer registries can account for Latino heterogeneity in their analyses. State death certificates can supplement surveillance data (incidence and survival) in order to find Latinospecific group and place of birth-key pieces of information needed to analyze Latino heterogeneity (Chap. 2: Pinheiro, Callahan, and Kobetz; Chap. 5: Stern).

\section{Cancer Risk, Prevention, and Screening}

- Consider heterogeneity among Latinas when estimating breast cancer risk and mortality rates. There is evidence that both the risk of developing breast cancer and mortality rate varies among and within Latino subgroups based upon country of origin, nativity, and genetic ancestry (Chap. 4: Fejerman, SerranoGómez, and Tamayo).

- Support international collaborations to access population data and biorepositories in Latin America. Acquisition of these data will enrich the study of the molecular diversity of cancer in Latinos, helping researchers to better understand cancer risk and outcomes related to biological, environmental, cultural, and access-related factors in individuals of Latin American origin (Chap. 4: Fejerman, Serrano-Gómez, and Tamayo).

- Conduct more epidemiological studies on prostate cancer using adequate risk factor data that includes Latinos. Prostate cancer (PCa) is the top cancer 
that affects Latino men, yet few Latinos have been included in epidemiological studies in the United States. Also needed are analyses of genetic ancestry and PCa risk among Latinos, which may inform some of the cancer incidence findings; and patterns of tumor localization must still be characterized to improve PCa detection among Latinos. Finally, patterns of care and adherence to active surveillance must be studied to design culturally-sensitive interventions (Chap. 5: Stern).

- Consider ethnic and cultural background in the design of research and interventions to improve diet among Latino groups. Most Latino groups fall short of American Cancer Society recommendations for the ideal preventative diet. Incorporate cultural dietary patterns and emphasize traditional healthy foods when designing nutritional interventions and research; this will help to reduce bias and establish reasonable portion sizes. Conduct longitudinal studies of diet and cancer outcomes among understudied Latino populations to reduce current cancer risk and prevent widening risk with the adoption of a Western diet (Chap. 6: Tucker).

\section{The Biology of Cancer Health Disparities}

- Find biomarkers associated with gastric pre-malignant lesions in Latinos. Gastric cancer disproportionately affects Latinos, and because early stage disease produces no symptoms, this cancer is often diagnosed as stage IV disease with low survival. It would be of great benefit to find biomarkers of treatable, pre-malignant stages and to devise strategies for the prediction of disease aggressiveness and outcome (Chap. 7: Garai, Li, and Zabaleta).

- Conduct comprehensive tumor genomic studies of gastric cancer in Latinos. Research suggests that the molecular profiles of gastric cancer in Latinos are unique. Thus, there is a need to characterize genetic and genomic patterns of gastric cancer in patients of Latino ancestry and to conduct preclinical and translation studies in driver genes and molecular subtypes that are more prevalent in Latinos (Chap. 8: Carvajal-Carmona).

- Elucidate biological factors that promote outcome disparities among Latinas with breast cancer. Breast cancer in Latinas develops at a younger age, and there is evidence that this early onset disparity may result from genetic, environmental, and biological factors such as altered estrogen metabolism resulting from childhood obesity (Chap. 9: Colon-Otero).

- Investigate the role of ethnicity in breast cancer susceptibility. Differences in gene expression profiles of breast cancer may be a consequence of ancestry and thus may be useful for studying breast cancer susceptibility in Latinos. Researchers must properly classify genetic ancestry in admixed Latino populations not only to understand the role of genetics in disease susceptibility, but also to provide Latinos the benefits of recent treatment advances (Chap. 10: SerranoGomez and Zabaleta). 


\section{Advances in Cancer Therapy and Clinical Trials}

- Structure diverse ethnic patient participation in biomarker trials. This will provide the most comprehensive ability to apply data to the general population. One important issue is determining the sufficient number of diverse patients to adequately do the analysis (Chap. 11: Perez).

- Include biomarker-negative patients in clinical trials. Limiting a study to marker-positive patients may characterize a drug/diagnostic poorly. Sometimes researchers incorrectly assume that a biomarker is required for a response and may thus deny effective therapy to marker-negative patients (Chap. 11: Perez).

- Improve Latino recruitment into cancer clinical trials. Latinos are underrepresented in cancer research, data registries, and genomic databases; this disparity can be addressed by improving Latino accrual into clinical trials. Suggestions are to educate physicians to promote enrollment, build awareness among Latinos about the role of clinical trials in improving cancer care, enhance care navigation for treatment planning; and develop language- and culture-appropriate educational materials (Chap. 12: Mesa and Ramirez).

- Use comorbidities as exclusion criteria only for sound evidence-based reasons. One of the barriers to Latino accrual in clinical trials is the use of comorbidities such as hypertension and obesity. Carefully determine if these exclusion criteria are necessary and evidence-based (Chap. 21: Croyle).

- Attract more Latinos into the pipeline for higher education in the health professions and medical school. Provide opportunities for Latinos to become health professionals or STEM researchers of the future. Try to attract more Latinos into the pipeline for higher education and medical school (Chap. 12: Mesa and Ramirez; Chap. 21: Rios).

\section{Cancer in the Era of Precision Medicine}

- Include more Latinos in precision medicine research. Genomic and transcriptomic studies are based primarily on tumors from Americans of northern European ancestry. Without adequate representation of patient diversity, precision medicine based on these studies may actually worsen health disparities (Chap. 13: Zabaleta et al.).

- Consider ethnicity and genetic ancestry when making cancer treatment choices based on gene expression profiles. For example, luminal breast cancers in Latinos may have distinctive biology due to non-genetic and/or ancestrylinked factors (Chap. 13: Zabaleta et al.).

- Ensure that Latinos have access to tumor molecular analysis for targeted cancer therapy. Targeted therapy is recommended as standard of care for colorectal and thyroid cancers. Compared to non-Latino whites, Latinos have 
higher incidence rates of these two cancers, so it is crucial that they have access to tumor molecular analysis (Chap. 14: Rodriguez-Rodriguez).

\section{Cancer Outcomes and Survivorship in Latinos}

- Provide access to cancer survivorship care that is linguistically and culturally appropriate for Spanish-speaking Latinos. Engage Latino communities in design and implementation of behavioral interventions that can be delivered in community settings and are linked to cancer care systems. Investigators can integrate evidence-based programs with community knowledge and best practices to test and disseminate co-developed programs to meet the needs of vulnerable cancer survivors (Chap. 15: Nápoles).

- Engage multiple stakeholders in patient-centered outcomes research on intervention design. Research democracy, where all stakeholders-including patients and their caregivers - have a voice and a vote in research decisions, can improve patient-centered outcomes research (PCOR) by promoting team engagement, satisfaction, and awareness of project barriers and benefits (Chap. 16: Kuo et al.).

\section{Engaging Latinos in Cancer Research}

- Engage Latinos in cancer prevention and control research. Community-level interventions that use community-based participatory research principles and that engage Latinos from the beginning of the research through the dissemination of data have the potential to reduce cancer disparities and save lives. Latina involvement can help researchers develop interventions that include social and cultural assets-essential elements that can help to eliminate disparities in cancer screening, prevention, and control (Chap. 17: Baezconde-Garbanati et al.).

- Deliver personalized interventions through texting and mobile media services. This scalable service makes mobile, personalized advice/support affordable; it can reach disadvantaged populations, produce a public health impact, reduce health service costs, and reduce health disparities in Latinos. Effectively used for smoking cessation, text messaging is interactive and can be delivered anonymously wherever the person is located (Chap. 18: Chalela et al.).

- Design cervical cancer interventions that target cultural factors which may operate at various levels of influence. Also, broaden the focus of interventions beyond cancer screening to improved sexual and reproductive health. Such interventions can target root causes of disparities and yield sustainable behavior change (Chap. 19: Lechuga and Melo).

- Use a community-based approach to increase participation of Latinos in biobanking and biospecimen research. Latinos are underrepresented in 
biomedical research and biospecimen banks. One solution is to partner with Latino communities in a culturally appropriate manner; deliver communitybased education programs to increase awareness and informed decision-making about biospecimen donation; and use mobile lab units to collect and process biospecimen donations onsite (Chap. 20: Rodriguez and Erwin).

\section{Emerging Policies in US Health Care}

- Researchers who study cancer in Latinos must advocate to Congress for their own work and educate policy makers of its value in helping people and saving lives. Researchers should be persistent in this effort, because lawmakers deal with many issues in addition to health care and may need reminding to refocus their attention (Chap. 21: Castro, Rios).

- Protect the Affordable Care Act. More Latinos than any other group were able to receive insurance coverage because of that act, so researchers and community members must make sure that it is not completely eroded (Chap. 21: Castro and Rios).

- Address the health effects of environmental quality on cancer and other illnesses. Some Latino communities are exposed to damaging environmental factors because of weak enforcement in certain states. Be mindful not only of Congress and the federal government but also of state and local jurisdictions (Chap. 21: Castro).

- Empower non-Latino physician partners with cultural competence to enhance their relationship with Latino patients and their caregivers. Only a small percentage of physicians are Latino, and the primary care physician can be the number one driver or barrier for proper cancer care and adherence to treatment (Chap. 21: López and Croyle).

- Foster survivor advocacy within the community. Involve Latino cancer survivors in advocacy, because the powerful voice of survivors can be leveraged to motivate Latinos to seek cancer screening (Chap. 21: López).

- Leverage online channels. The Internet is a powerful channel for Latinos to find information and additional support along the path to treatment (Chap. 21: López).

- Protect patients through local policy. Physicians, health care systems, and insurance companies must advocate for the communities they serve. Latinos treated for chronic conditions often experience higher incidence of complication and death than their non-Latino counterparts, so advocacy is important (Chap. 21: López). 


\section{Charting the Future of Cancer Health Disparities Research in Latinos}

The purpose of this conference was to move beyond documented health disparities in Latinos to set a new agenda for future initiatives that specifically address cancer health disparities and improve outcomes in Latino communities. At the close of the conference, Drs. Trapido (LSU Stanley Scott Cancer Center), Cruz-Correa (University of Puerto Rico Comprehensive Cancer Center), and Stern (University of Southern California) moderated a session entitled Charting the Future of Cancer Health Disparities Research in Latinos. The group determined research priority areas based upon the papers presented at the meeting. Attendees were asked to respond to a live poll and select up to three areas they considered top priorities for cancer research in Latinos.

The top five priority areas for cancer research in Latinos for Parts II, III, and IV were ranked from one to five as follows:

1. Larger cohort studies on genetics vs. environment in Latinos to understand health differences.

2. Translate effective lifestyle programs to fit the community context and population.

3. Multinational studies comparing native born in countries of origin vs. United States.

4. Investigate ancestry and infectious agents which cause cancer.

5. Develop and implement interventions for sexual health (i.e., assessment of outcomes, longitudinal designs, standardization).

The top five priority areas for cancer research in Latinos for Parts V, VI, and VII were:

1. Reduce barriers of enrollment for Latinos in clinical trials and include patient reported outcomes and ethnic diversity.

2. Link databases to catalyze biomarker precision medicine and precision oncology.

3. Examine the relationship of genes/pathways to determine metaplasia that might progress to cancer, as well as SNPs in key genes among Latinos.

4. Mitigate the impact of trial globalization on the relevance of medical products/ drugs to the Latino community.

5. Evaluate pathways in carcinogenesis in $\mathrm{HCC}$ to explore therapies.

The top five priority areas for cancer research in Latinos for Parts VIII and IX were:

1. Conduct long-term follow up in intervention studies.

2. Include more family system-based approaches.

3. Improve integration across disciplines. 
4. Get Latinas and other minorities to participate in cancer research and pay it forward.

5. Measure depression better in Latinos.

This publication describes the beginning of much needed research tailored to improve understanding of key contributors to cancer in the Latino population. Recommendations from the conference participants give us a direction for future research that will advance the science of cancer in Latinos and eventually lead to lives saved. And until we reach populations with needed screening, treatment, and improved quality of life for cancer survivors, we will not see a decline in cancer deaths. Reducing the cancer burden in Latino communities takes all of us working together throughout the continuum of cancer.

\section{References}

1. Ramirez AG, Talavera GA, Marti J, Penedo FJ, Medrano MA, Giachello AL, et al. Redes En Accion. Increasing Hispanic participation in cancer research, training, and awareness. Cancer. 2006;107(8 Suppl):2023-33. https://doi.org/10.1002/cncr.22148.

2. Ramirez AG, Gallion KJ, Suarez L, Giachello AL, Marti JR, Medrano MA, et al. A national agenda for Latino cancer prevention and control. Cancer. 2005;103(11):2209-15. https://doi. org/10.1002/cncr.21053.

3. Ramirez AZ, McAlister AL, Villarreal R, Suarez L, Talavera GA, Pérez-Stable EJ, et al. Prevention and control in diverse Hispanic populations: a national initiative for research and action. Cancer. 1998;83(8 Suppl):1825-9.

4. Ramirez AG, Perez-Stable EJ, Talavera GA, Penedo FJ, Carrillo JE, Fernandez ME, et al. Time to definitive diagnosis of breast cancer in Latina and non-Hispanic white women: the six cities study. Springerplus. 2013;2(1):84. https://doi.org/10.1186/2193-1801-2-84.

Open Access This chapter is licensed under the terms of the Creative Commons AttributionNonCommercial 4.0 International License (http://creativecommons.org/licenses/by-nc/4.0/), which permits any noncommercial use, sharing, adaptation, distribution and reproduction in any medium or format, as long as you give appropriate credit to the original author(s) and the source, provide a link to the Creative Commons license and indicate if changes were made.

The images or other third party material in this chapter are included in the chapter's Creative Commons license, unless indicated otherwise in a credit line to the material. If material is not included in the chapter's Creative Commons license and your intended use is not permitted by statutory regulation or exceeds the permitted use, you will need to obtain permission directly from the copyright holder.

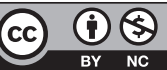

